\title{
GESTÃO DO RISCO CARDIOVASCULAR EM PESSOAS COM DOENÇA CRÓNICA NA COMUNIDADE: UMA REVISÃO SISTEMÁTICA DA LITERATURA
}

\author{
Helga Rafael Henriques ${ }^{1}$, Joana Pinto², José Faria ${ }^{2}$, Augusto Silva ${ }^{3}$ \\ ${ }^{1}$ Escola Superior de Enfermagem de Lisboa, Portugal. hrafael@esel.pt; \\ ${ }^{2}$ Centro Hospitalar Universitário de Lisboa Central e ESEL- Lisboa, Portugal. joanapinto@esel.pt; jose.faria@esel.pt \\ ${ }^{3}$ Centro Hospitalar Lisboa Norte, Portugal. gusto.silva83@gmail.com
}

Resumo. As doenças cardiovasculares (DCV) são a principal causa de morte em todo o mundo. Os seus fatores de risco sobrepõem-se aos das principais doenças crónicas. Existe evidência insuficiente no que respeita à efetividade de intervenções de promoção da gestão do risco cardiovascular na doença crónica, pelo que a investigação sobre novas formas de cuidados de saúde é uma prioridade. Temos como objetivo identificar os componentes dos programas de gestão do risco cardiovascular, benefícios e técnicas de mudança comportamental utilizadas. Realizou-se uma revisão sistemática da literatura nas bases de dados Scopus, MEDLINE, CHINAL, Cochrane Central Register of Controlled Trials, e Medlatina. Obteve-se uma amostra bibliográfica de 16 artigos, envolvendo 20301 pessoas com doença crónica. Os programas de intervenção integram, de forma isolada ou combinada, os elementos educação para a saúde, exercício físico, alimentação, vigilância de saúde e psicologia motivacional. Revelam-se heterogéneos nos seus componentes, duração e estratégias utilizadas. Recorrem à intervenção uni ou multidisciplinar. Concluímos que os resultados indicam algumas intervenções promissoras na gestão do risco de DCV em pessoas com doenças crônicas. No entanto, a heterogeneidade dos programas e a inconsistência de alguns resultados impedem conclusões pouco sólidas. É necessário continuar a investigar para tornar os resultados mais consistentes.

Palavras-chave: Doença Crónica; Autogestão; Gestão do Risco; Programa

\section{MANAGING CARDIOVASCULAR RISK IN PEOPLE WITH CHRONIC DISEASE IN THE COMMUNITY: A SYSTEMATIC REVIEW OF THE LITERATURE}

\begin{abstract}
Cardiovascular diseases (CVD) are the leading cause of death worldwide. Its risk factors overlap with those of the main chronic diseases. There is insufficient evidence regarding the effectiveness of interventions to promote cardiovascular risk management in chronic disease, so research into new forms of health care is a priority. We aim to identify the components of cardiovascular risk management programs, benefits and behavioral change techniques used. A systematic review of the literature was carried out in the Scopus, MEDLINE, CHINAL, Cochrane Central Register of Controlled Trials, and Medlatina databases. We found a bibliographic sample of 16 articles, involving 20301 people with chronic disease. The intervention programs integrate, in an isolated or combined way, the elements health education, physical exercise, food, health surveillance and motivational psychology. They are heterogeneous in their components, duration and strategies used. They resort to uni or multidisciplinary intervention. We conclude that the results indicate some promising interventions in the management of CVD risk in people with chronic diseases. However, the heterogeneity of the programs and the inconsistency of some results prevent poor conclusions. Further research is needed to make the results more consistent.
\end{abstract}

Keywords: Chronic Disease; Self-Management; Risk Management; Program 


\section{INTRODUÇÃO}

As doenças cardiovasculares (DCV) são a principal causa de morte em todo o mundo, representando $44 \%$ das mortes por doenças crónicas não transmissíveis (WHO, 2018). Estima-se que 17,9 milhões de pessoas morreram de DCV em 2016, representando 31\% de todas as mortes globais. As DCV são um grupo de enfermidades que incluem as doenças do coração e dos vasos, sendo as mais frequentes (85\%), o enfarte agudo do miocárdio e o acidente vascular cerebral (WHO, 2017).

Em Portugal, a mortalidade por doenças do aparelho circulatório contrariou a tendência decrescente entre 2013 e 2014, ultrapassando os 30\% (DGS, 2016). Este acréscimo deveuse ao aumento das mortes associadas a doença isquémica cardíaca. Na sequência destes dados, o Programa Nacional para as Doenças Cérebro-cardiovasculares (DGS, 2016) modificou as suas metas e objetivos estratégicos, passando a eleger como prioritária a redução da mortalidade prematura ( $<70$ anos).

A maioria das doenças cardiovasculares pode ser evitada através de uma adequada gestão dos fatores de risco comportamentais, como uso de tabaco, dieta não saudável e obesidade, inatividade física e uso nocivo de álcool (WHO, 2017). A evidência mais recente mostra existir uma ligação muito forte entre os fatores de risco ligados ao estilo de vida, anteriormente descritos, e o risco de doenças não transmissíveis (Peters et al., 2019), sendo que estes coincidem com os das DCV.

De uma forma isolada, alguns estudos mostram a efetividade de uma ou duas áreas de intervenção na gestão do risco de DCV. Assim, a atividade física é eficaz para a prevenção e tratamento de doenças crónicas, como a DCV, o cancro, a diabetes e as doenças respiratórias crónicas, podendo os programas domiciliares ser tão viáveis quanto os programas de atividade física supervisionados por clínicos (Bullard, Ji, An, Trinh, Mackenzie \& Mullen, 2019). Da mesma forma, alterações positivas no estilo de vida, como o aumento da atividade física e mudanças na alimentação, têm sido consideradas fulcrais no controlo da hipertensão e pré-diabetes (Pengpid, Peltzer, Puckpinyo, \& Chantarasongsuk, 2019).

A gestão do risco cardiovascular pressupõe a implementação de intervenções de promoção da saúde, dirigidas a mais do que um fator de risco ao mesmo tempo. Contudo, a evidência disponível nem sempre investiga a implementação destas intervenções de forma conjugada, 
o que não permite concluir acerca da sua eficácia sobre a gestão do risco de DCV (Uthman et al., 2015).

De acordo com o Chronic Care Model (Wagner, 2001), a gestão da doença crónica inclui dimensões como a organização dos cuidados de saúde, uma rede de serviços, suporte à tomada de decisão, sistema de informação clínica, suporte à autogestão e recursos comunitários. Contudo, poucos são os programas de promoção da gestão da doença crónica que abordam todos estes componentes. O suporte na autogestão da doença crónica é a intervenção com resultados mais significativos (Reynolds et al., 2018), havendo evidência de que o cuidado colaborativo pode trazer resultados estatisticamente significativos e positivos em pessoas com múltiplas doenças crónicas (Luijks et al., 2015; Kastner et al., 2018).

Entendemos a autogestão de doenças crónicas como a intervenção sistemática, direcionada à pessoa com estas problemáticas, que the permite participar ativamente na automonitorização (de sintomas ou de processos fisiológicos) e na tomada de decisão (gestão da doença e seu impacto) (Chodosh et al., 2005).

As técnicas de mudança de comportamento são essenciais para o desenho e implementação de programas promotores da autogestão da doença crónica. Uma técnica de mudança de comportamento é um elemento observável, replicável e irredutível de uma intervenção projetada para alterar ou redirecionar processos causais que regulam o comportamento, ou seja, propõe-se que uma técnica seja um 'ingrediente ativo' (Michie et al., 2013). Quisemos, portanto, perceber quais os componentes dos programas de gestão do risco cardiovascular, dando atenção às características das intervenções, benefícios e técnicas de mudança comportamental utilizadas.

A evidência disponível no que respeita à efetividade de intervenções de promoção da gestão da doença crónica é manifestamente insuficiente, pelo que a investigação sobre novas formas de cuidados de saúde é uma prioridade (Xu, Mishra, \& Jones, 2017; Van der Heide, 2018).

\section{METODOLOGIA}

O objetivo deste estudo foi identificar os componentes de um programa de gestão do risco cardiovascular, na população adulta com doença crónica na comunidade. 
Para tal realizou-se uma revisão sistemática da literatura de acordo com os referenciais da PRISMA (Moher et al., 2015).

A pesquisa foi realizada nas bases de dados Scopus, MEDLINE, CHINAL, Cochrane Central Register of Controlled Trials, e Medlatina, num intervalo temporal entre 2015 e 2019.

A estratégia de pesquisa combinou os termos relacionados com a população (Chronic Disease, Noncommunicable Diseases), os termos relacionados com a intervenção (Program Development, program, Long-Term Care, or Community Health Planning) e os resultados esperados (Risk, Health Risk Behaviors, Risk Management, Risk Factors, Cardiovascular Diseases).

Os estudos identificados foram analisados por três revisores independentes.

Foram incluídos todos os estudos cujos participantes eram pessoas com mais de 18 anos, com doença crónica, em contexto comunitário, que tinham sido submetidas a um programa de intervenção promotor da gestão do risco cardiovascular.

Selecionaram-se ensaios clínicos aleatorizados, tendo sido possível comparar pessoas com doença crónica que receberam um programa de intervenção com pessoas com doença crónica que receberam os cuidados convencionais.

Os estudos foram rastreados pelo título e, em seguida, analisaram-se os resumos que forneceram informações mais precisas sobre a população ou intervenção utilizada.

Foram excluídos os estudos cujos participantes eram profissionais de saúde, pessoas com doenças transmissíveis, crianças, grávidas ou puérperas. Excluíram-se também estudos qualitativos, artigos de opinião, protocolos de investigação e revisões da literatura (Figura 1). 


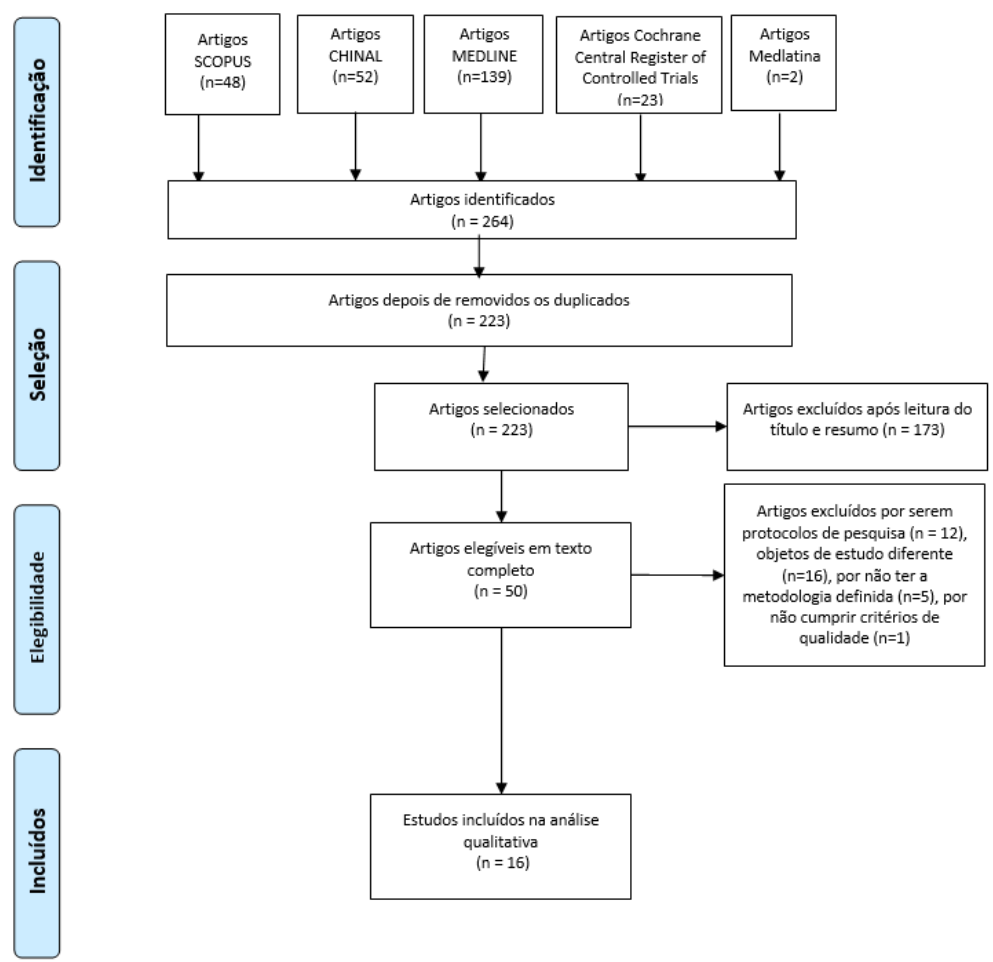

Figura 1. Diagrama da Revisão Sistemática da Literatura

Para garantir a qualidade dos estudos, foram incluídos, estudos experimentais aleatorizados (12), estudos quasi experimental (2), estudo coorte (1) e estudo epidemiológico retrospetivo (1), de acordo com os níveis de qualidade metodológica (Meader, et al., 2014).

A análise dos estudos decorreu tendo por base o Chronic Care Model (Wagner, 2001) e as técnicas de mudança de comportamentos propostas por Michie, Atkins \& West (2014).

\section{RESULTADOS E DISCUSSÃO}

Do processo de seleção dos artigos resultou uma amostra bibliográfica de 16 artigos, envolvendo 20301 pessoas com doença crónica, de países como EUA, Canadá, Austrália, China, República da Coreia, Irão, Itália, Taiwan e Alemanha. Os estudos focavam-se em apenas uma morbilidade, ou em multimorbilidades, estando descritas as seguintes patologias obesidade (4), dislipidemia (3), HTA (4), diabetes (8) e doenças cardiovasculares (4). Dos artigos selecionados extraíram-se os dados que constam no quadro seguinte (Quadro 1). 
Quadro 1. Estudos extraídos

\begin{tabular}{|c|c|c|c|}
\hline $\begin{array}{l}\text { Código/ } \\
\text { Estudo }\end{array}$ & Objetivo & $\begin{array}{l}\text { Componentes da } \\
\text { intervenção }\end{array}$ & Resultados \\
\hline $\begin{array}{l}1 \\
\text { Abbott et al., } \\
2020\end{array}$ & $\begin{array}{l}\text { Determinar o efeito de } \\
\text { um programa de } \\
\text { promoção da saúde } \\
\text { sobre o conhecimento } \\
\text { de diabetes e } \\
\text { comportamentos de } \\
\text { autocuidado } \\
\text { autorrelatados }\end{array}$ & $\begin{array}{l}\text { Sessões de educação em } \\
\text { grupo sobre diabetes }\end{array}$ & $\begin{array}{l}\text { O grupo de intervenção teve mudanças } \\
\text { significativas, do pré-teste ao pós-teste, nos } \\
\text { conhecimentos sobre diabetes e comportamentos } \\
\text { relacionados com a dieta e testes de glicose no } \\
\text { sangue }\end{array}$ \\
\hline $\begin{array}{l}5 \\
\text { Al Hamarneh } \\
\text { et al, } 2017\end{array}$ & $\begin{array}{l}\text { Avaliar o efeito de um } \\
\text { programa de } \\
\text { intervenção de } \\
\text { farmacêuticos na } \\
\text { determinação do risco } \\
\text { cardiovascular em } \\
\text { pessoas com diabetes }\end{array}$ & $\begin{array}{l}\text { Consultas realizada por um } \\
\text { farmacêutico a cada } 4 \\
\text { semanas, que incluíam } \\
\text { avaliação da pessoa, } \\
\text { avaliação laboratorial, } \\
\text { avaliação individualizada do } \\
\text { risco cardiovascular. } \\
\text { Sempre que identificadas } \\
\text { alterações pressupunha } \\
\text { comunicação com o médico } \\
\text { assistente }\end{array}$ & $\begin{array}{l}\text { O risco cardiovascular estimado reduziu em maior } \\
\text { grau no grupo de intervenção }\end{array}$ \\
\hline $\begin{array}{l}8 \\
\text { Blackford et } \\
\text { al., } 2016\end{array}$ & $\begin{array}{l}\text { Avaliar a eficácia de } \\
\text { uma intervenção para } \\
\text { pessoas adultas rurais } \\
\text { com, ou sem risco de } \\
\text { síndrome metabólico, } \\
\text { baseada em atividade } \\
\text { física e dieta }\end{array}$ & $\begin{array}{l}\text { Exercício físico em casa e } \\
\text { aconselhamento nutricional }\end{array}$ & $\begin{array}{l}\text { O grupo de intervenção melhorou } \\
\text { significativamente o seu perfil de triglicéridos, } \\
\text { colesterol total e o colesterol não-HDL em } \\
\text { comparação com o grupo de controlo. O perímetro } \\
\text { abdominal, razão cintura-anca e o índice de } \\
\text { massa corporal também melhoraram }\end{array}$ \\
\hline $\begin{array}{l}13 \\
\text { Chao et al., } \\
2017\end{array}$ & $\begin{array}{l}\text { Avaliar a eficácia de } \\
\text { um aplicativo móvel } \\
\text { (WeChat), na perda } \\
\text { de peso }\end{array}$ & Educação alimentar & $\begin{array}{l}\text { Os participantes no grupo WeChat perderam mais } \\
\text { peso do que as pessoas no grupo de controlo. Os } \\
\text { participantes do sexo masculino mais ativos foram } \\
\text { usando o WeChat, os que perderam mais peso. } \\
\text { Pode não haver nenhum efeito ou pode até haver } \\
\text { um efeito negativo sobre a perda de peso para as } \\
\text { mulheres. }\end{array}$ \\
\hline $\begin{array}{l}19 \\
\text { Kim et al., } \\
2019\end{array}$ & $\begin{array}{l}\text { Investigar o efeito do } \\
\text { exercício de } \\
\text { reabilitação na saúde } \\
\text { vascular }\end{array}$ & $\begin{array}{l}\text { Programa de reabilitação, } \\
\text { com utilização de exercícios } \\
\text { de alongamento ativo- } \\
\text { passivo }\end{array}$ & $\begin{array}{l}\text { Existem efeitos de interação significativos no } \\
\text { índice braquial do tornozelo (ITB) e no índice } \\
\text { vascular cardio-tornozelo (CAVI). O programa de } \\
\text { reabilitação mostrou ter um efeito positivo na } \\
\text { saúde vascular e acreditasse ser uma medida } \\
\text { preventiva eficaz para reduzir a incidência e o } \\
\text { risco de doença cardiovascular. }\end{array}$ \\
\hline $\begin{array}{l}20 \\
\text { Fitzpatrick et } \\
\text { al., } 2016\end{array}$ & $\begin{array}{l}\text { Comparar a eficácia } \\
\text { de três modalidades } \\
\text { de ensino no } \\
\text { processo de tomada } \\
\text { de decisão quotidiano } \\
\text { na pessoa com } \\
\text { diabetes tipo } 2 \\
\text { (programa DECIDE) }\end{array}$ & $\begin{array}{l}\text { Educação para a saúde em } \\
\text { sessões individuais, } \\
\text { sessões de grupo e estudo } \\
\text { autónomo }\end{array}$ & $\begin{array}{l}\text { As modalidades DECIDE e o grupo controlo não } \\
\text { diferiram significativamente nos resultados clínicos } \\
6 \text { meses após a intervenção. Nos participantes } \\
\text { com A1C } \geq 7,5 \% \text { inicialmente, baixou nas várias } \\
\text { modalidades DECIDE na primeira semana pós- } \\
\text { intervenção. Apenas no estudo autónomo aos } 6 \\
\text { meses após a intervenção houve redução } \\
\text { significativa da pressão arterial sistólica. No } \\
\text { estudo autónomo e no individual houve declínio } \\
\text { significativo no LDL. No estudo autónomo houve } \\
\text { um aumento no HDL aos } 6 \text { meses após a } \\
\text { intervenção. O estudo autónomo e o individual } \\
\text { tiveram um aumento maior no conhecimento do } \\
\text { que o grupo de controle. Todos melhoraram na } \\
\text { resolução de problemas } 6 \text { meses após a } \\
\text { intervenção. }\end{array}$ \\
\hline $\begin{array}{l}23 \\
\text { Hung et al., } \\
2016\end{array}$ & $\begin{array}{l}\text { Determinar se um } \\
\text { programa de } \\
\text { telessaúde, de quarta } \\
\text { geração, pode reduzir } \\
\text { a mortalidade por } \\
\text { todas as causas em }\end{array}$ & $\begin{array}{l}\text { Auto monitorização, } \\
\text { acompanhamento } \\
\text { telefónico de enfermagem e } \\
\text { consulta médica }\end{array}$ & $\begin{array}{l}\text { O programa de telessaúde de quarta geração está } \\
\text { associado a menor mortalidade por todas as } \\
\text { causas, em comparação com os cuidados usuais } \\
\text { após o ajuste para comorbidades crónicas. } \\
\text { Os resultados sugerem que o efeito benéfico da } \\
\text { telessaúde aumenta ao longo do tempo. }\end{array}$ \\
\hline
\end{tabular}




\begin{tabular}{|c|c|c|}
\hline $\begin{array}{l}\text { Código/ } \\
\text { Estudo }\end{array}$ & Objetivo & $\begin{array}{l}\text { Componentes da } \\
\text { intervenção }\end{array}$ \\
\hline & $\begin{array}{l}\text { pessoas com doença } \\
\text { cardiovascular crónica }\end{array}$ & \\
\hline $\begin{array}{l}24 \\
\text { Kempf et al., } \\
2017\end{array}$ & $\begin{array}{l}\text { Avaliar a eficácia de } \\
\text { um programa de } \\
\text { intervenção no Estilo } \\
\text { de Vida Telemedical } \\
\text { (TeLiPro), na melhoria } \\
\text { do controle } \\
\text { metabólico em } \\
\text { pessoas com diabetes } \\
\text { tipo } 2 \text { em estadio } \\
\text { avançado }\end{array}$ & $\begin{array}{l}\text { Sessões de educação em } \\
\text { grupo, intervenção } \\
\text { nutricional, consulta médica } \\
\text { e atendimento telefónico. }\end{array}$ \\
\hline
\end{tabular}

Resultados

A redução da $\mathrm{HbA} 1 \mathrm{c}$ foi significativamente maior no grupo TeliPro. Na diabetes tipo 2 em estadio avançado, o TeLiPro pode melhorar o controle glicémico e pode oferecer novas opções para evitar a intensificação farmacológica.

$\begin{array}{ll} & \text { Avaliar os efeitos de } \\ & \text { uma dieta com } \\ & \text { restrição de energia, } \\ & \text { com e sem } \\ & \text { deambulação } \\ & \text { moderada, no peso } \\ 26 & \text { corporal, composição } \\ \text { Kleist et al., } & \text { corporal, gasto } \\ 2017 & \text { energético de repouso } \\ & \text { (GER) e variáveis de } \\ & \text { risco endócrino e } \\ & \text { cardiometabólico em } \\ & \text { pessoas com excesso } \\ & \text { de peso e obesidade }\end{array}$

\begin{tabular}{|c|c|c|}
\hline $\begin{array}{l}27 \\
\text { Koniak- } \\
\text { Griffin et al., } \\
2015\end{array}$ & $\begin{array}{l}\text { Avaliar a viabilidade } \\
\text { de uma intervenção } \\
\text { nos estilos de vida, } \\
\text { em mulheres latinas e } \\
\text { imigrantes com } \\
\text { excesso de peso }\end{array}$ & $\begin{array}{l}\text { Educação para a saúde } \\
\text { sobre estilos de vida e } \\
\text { acompanhamento } \\
\text { presencial e via telefónica }\end{array}$ \\
\hline $\begin{array}{l}29 \\
\text { Malagoni et } \\
\text { al., } \\
2016\end{array}$ & $\begin{array}{l}\text { Verificar a viabilidade, } \\
\text { segurança e eficácia } \\
\text { preliminar de um } \\
\text { modelo original de } \\
\text { reabilitação em casa, } \\
\text { comparado a um } \\
\text { programa } \\
\text { supervisionado } \\
\text { padrão em } \\
\text { sobreviventes de AVC } \\
\text { hemiplégico crónico }\end{array}$ & $\begin{array}{l}\text { Exercícios músculo- } \\
\text { esqueléticos e caminhada } \\
\text { no domicílio }\end{array}$ \\
\hline
\end{tabular}

Avaliar a viabilidade e eficácia de uma 30 intervenção de Marra et al., telenutrição nos 2019 cuidados saúde primários para perda de peso
Caminhada ao ar livre

(supervisionada e autónoma) e intervenção nutricional
Educação nutricional e acompanhamento via telefónico
Existiram reduções significativas no peso corporal e massa gorda no grupo de intervenção, que foi associado a melhorias significativas nos biomarcadores para risco de doença cardiovascular.

A perda moderada de peso não foi acompanhada de uma redução no gasto energético de repouso. Caminhadas moderadas adicionais aumentaram os efeitos de uma dieta hipocalórica na perda de gordura e na redução da insulinoresistência. A pressão arterial diastólica, pressão arterial média, colesterol LDL e colesterol HDL também foram significativamente melhoradas pelas duas intervenções. A triiodotironina livre diminuiu significativamente e o cortisol sérico aumentou significativamente em ambos os grupos.

Esta intervenção trouxe melhorias significativas nos biomarcadores para redução do risco de doença cardiovascular.

Os comportamentos de estilo de vida e outros fatores de risco de mulheres latinas com excesso de peso podem ser melhorados através de uma intervenção comportamental de alteração de estilos de vida.

O teste de marcha de 6 minutos e o domínio de atividade física aumentaram significativamente nos dois grupos. O tempo necessário para os profissionais de saúde implementarem o programa de exercícios em casa foi de 15horas VS 30horas para o programa supervisionado padrão. O programa estruturado de exercícios em casa é viável, seguro e capaz de induzir melhorias na capacidade funcional e na qualidade de vida comparando com um programa convencional de reabilitação supervisionado

Ambos os grupos reduziram o peso corporal, perímetro abdominal, percentagem de gordura, ingestão calórica e melhoraram a qualidade da dieta. Verificou-se maior perda de peso no grupo experimental.

As taxas de retenção, adesão e satisfação relatadas pelos participantes foram $\geq 80 \%$ no grupo de telenutrição. As intervenções de telenutrição nos cuidados saúde primários têm o potencial de melhorar o acesso ao aconselhamento dietético para tratamento da obesidade

A distância do teste de marcha melhorou em $33 \quad$ Avaliar o efeito do Mills et al., programa Work It Out 2017 nos resultados média $0,053 \mathrm{~km}$, influenciada pelas condições sociais e emocionais. As pessoas com obesidade 


\begin{tabular}{|c|c|c|c|}
\hline $\begin{array}{l}\text { Código/ } \\
\text { Estudo }\end{array}$ & Objetivo & $\begin{array}{l}\text { Componentes da } \\
\text { intervenção }\end{array}$ & Resultados \\
\hline & $\begin{array}{l}\text { clínicos em pessoas } \\
\text { citadinas de } \\
\text { Aboriginal and Torres } \\
\text { Strait Islander com ou } \\
\text { sem risco de doença } \\
\text { cardiovascular }\end{array}$ & & $\begin{array}{l}\text { extrema diminuíram em média 1,6kg o peso } \\
\text { corporal. } \\
\text { Os participantes com pressão arterial sistólica } \\
\text { basal alta demonstraram uma diminuição média } \\
\text { de } 11 \mathrm{mmHg} \text {. } \\
\text { O programa Work It Out pode ser um modelo útil } \\
\text { para reabilitação e prevenção doença } \\
\text { cardiovascular para as populações citadinas }\end{array}$ \\
\hline $\begin{array}{l}45 \\
\text { Rigamonti et } \\
\text { al., } 2019\end{array}$ & $\begin{array}{l}\text { Investigar os efeitos } \\
\text { de um programa de } \\
\text { redução de peso } \\
\text { corporal. }\end{array}$ & $\begin{array}{l}\text { Dieta com restrição } \\
\text { calórica, educação } \\
\text { nutricional, aconselhamento } \\
\text { psicológico, atividade física; }\end{array}$ & $\begin{array}{l}\text { Redução de } 4 \% \text { no peso corporal foi alcançado no } \\
\text { final do programa. Foi associado a uma } \\
\text { diminuição do colesterol e glicose, e uma redução } \\
\text { da pressão arterial sistólica (redução do risco } \\
\text { cardiovascular). }\end{array}$ \\
\hline $\begin{array}{l}51 \\
\text { Teychenne } \\
\text { et al., } 2015\end{array}$ & $\begin{array}{l}\text { Comparar a eficácia } \\
\text { de um programa de } \\
\text { treino de força padrão } \\
\text { com um programa } \\
\text { personalizado na } \\
\text { adoção e manutenção } \\
\text { de fatores de risco e } \\
\text { cardiometabólicos e } \\
\text { força muscular. }\end{array}$ & $\begin{array}{l}\text { Programa de treino de força } \\
\text { e motivacional. }\end{array}$ & $\begin{array}{l}\text { Naqueles com DMT2, houve uma redução } \\
\text { significativa de HbA1c no programa adaptado } \\
\text { (grupo de intervenção) em comparação ao grupo } \\
\text { padrão (grupo controlo) durante a fase de } \\
\text { adaptação. }\end{array}$ \\
\hline $\begin{array}{l}54 \\
\text { Yazdanpana } \\
\text { h et al., } 2019\end{array}$ & $\begin{array}{l}\text { Determinar o efeito de } \\
\text { um programa } \\
\text { educacional baseado } \\
\text { no Modelo de } \\
\text { Crenças em Saúde } \\
\text { sobre adesão } \\
\text { medicamentosa em } \\
\text { idosos que sofrem de } \\
\text { hipertensão. }\end{array}$ & $\begin{array}{l}\text { Sessões educacionais em } \\
\text { grupo }\end{array}$ & $\begin{array}{l}\text { A adesão à medicação obtida pelo grupo de } \\
\text { intervenção foi significativamente superior ao } \\
\text { grupo controle. }\end{array}$ \\
\hline
\end{tabular}

Os programas de intervenção são bastante heterogéneos na sua natureza, composição, duração e técnicas de mudança comportamental utilizadas. Podemos verificar que existem programas de gestão do risco de DCV que integram os componentes: educação para a saúde (sessões individuais ou em grupo), exercício físico, alimentação, regime medicamentoso, acompanhamento/aconselhamento e psicologia motivacional. A distribuição destes componentes nas diferentes dimensões do Chronic Care Model (Wagner, 2001) permite perceber que se concentram no reforço da rede de serviços, na criação de novos sistemas de informação e no suporte à tomada de decisão e à autogestão (Tabela 1).

Tabela 1. Dimensões do Chronic Care Model presentes nos estudos (adaptado de Wagner, 2001)

\begin{tabular}{lcc}
\hline \multicolumn{1}{c}{ Chronic Care Model } & Fi & Fr \\
\hline Organização dos cuidados de saúde & 5 & 31.25 \\
Rede de serviços & 13 & 81.25 \\
Suporte à tomada de decisão & 15 & 93.75 \\
Sistemas de informação & 13 & 81.25 \\
Suporte autogestão & 12 & 75 \\
Recursos comunitário & 4 & 25 \\
\hline
\end{tabular}


A implementação de programas que se sustentam no Chronic Care Model mostrou ser efetiva na redução da utilização dos serviços de saúde e na redução do risco cardiovascular (Yeoh et al., 2018).

Os estudos analisados mostram que os diferentes componentes dos programas de gestão da doença crónica são implementados de forma isolada ou combinada, recorrendo a diferentes técnicas para promover a mudança comportamental (Quadro 2).

Quadro 2. Técnicas de mudança comportamental identificadas (adaptado de Behavior Change Technique Taxonomy (V1), de Michie, Atkins \& West, 2014)

\begin{tabular}{|c|c|c|}
\hline $\begin{array}{l}\text { Técnicas de mudança } \\
\text { comportamental }\end{array}$ & Definição & $\begin{array}{l}\text { Código dos } \\
\text { Estudos }\end{array}$ \\
\hline $\begin{array}{l}\text { 1. Objetivos e planeamento: } \\
\text { 1.1/1.3 Estabelecer objetivos } \\
\text { (comportamento/resultados) }\end{array}$ & $\begin{array}{l}\text { Negociar um objetivo em termos de (resultados) comportamento a } \\
\text { alcançar. }\end{array}$ & $\begin{array}{l}8,24,27,30 \\
45,51\end{array}$ \\
\hline 1.2 Resolução de problemas & $\begin{array}{l}\text { Analisar com a pessoa fatores que influenciam o comportamento e criar } \\
\text { estratégias para ultrapassar as barreiras ou potenciar os facilitadores. }\end{array}$ & $20,24,51$ \\
\hline 1.4 Planeamento da ação & $\begin{array}{l}\text { Definir detalhadamente um plano para } 0 \text { desempenho do } \\
\text { comportamento }\end{array}$ & $\begin{array}{l}24,26,29,30, \\
51\end{array}$ \\
\hline \multicolumn{3}{|l|}{ 2. Feedback e monitorização: } \\
\hline $\begin{array}{l}\text { 2.1 Monitorizar o } \\
\text { comportamento por outros }\end{array}$ & $\begin{array}{l}\text { Observa ou regista o comportamento com o conhecimento da pessoa } \\
\text { como uma estratégia de mudança comportamental }\end{array}$ & $5,13,23,45$ \\
\hline $\begin{array}{l}2.2 \text { Feedback sobre o } \\
\text { comportamento }\end{array}$ & Monitoriza e dá feedback sobre o desempenho do comportamento & $\begin{array}{l}13,23,30,45, \\
51\end{array}$ \\
\hline $\begin{array}{l}\text { 2.3/2.4 Automonitorização do } \\
\text { comportamento/resultados }\end{array}$ & $\begin{array}{l}\text { Estabelece um método para a pessoa monitorizar e registar o } \\
\text { comportamentos/resultados, como parte da estratégia de mudança } \\
\text { comportamental }\end{array}$ & $8,24,45$ \\
\hline \multicolumn{3}{|l|}{$\begin{array}{l}\text { 4. Modelando o } \\
\text { conhecimento }\end{array}$} \\
\hline $\begin{array}{l}4.1 \text { Instruir como desenvolver o } \\
\text { comportamento }\end{array}$ & $\begin{array}{l}\text { Aconselhar ou negociar formas de desenvolver o comportamento (inclui } \\
\text { treino de habilidades). }\end{array}$ & $\begin{array}{l}1,5,13,20,23 \\
24,26,27,30 \\
33,45,51,54\end{array}$ \\
\hline $\begin{array}{l}4.2 \text { Informação sobre } \\
\text { antecedentes }\end{array}$ & $\begin{array}{l}\text { Fornece informação sobre antecedentes que predizem o desempenho } \\
\text { do comportamento. }\end{array}$ & $1,23,24,26,45$ \\
\hline $\begin{array}{l}\text { 5. Consequências naturais } \\
5.1 \text { Informação sobre } \\
\text { consequências para a saúde }\end{array}$ & $\begin{array}{l}\text { Fornece informações sobre consequências para a saúde de } \\
\text { determinado comportamento. }\end{array}$ & $1,23,24$ \\
\hline $\begin{array}{l}\text { 6. Comparação de } \\
\text { comportamento } \\
6.1 \text { Demonstração do } \\
\text { comportamento }\end{array}$ & $\begin{array}{l}\text { Fornece um exemplo observável do comportamento desejado, } \\
\text { diretamente ou indiretamente, via filme ou imagem, para que a pessoa } \\
\text { se inspire ou imite. }\end{array}$ & $\begin{array}{l}8,19,24,26,27 \\
33,45,51\end{array}$ \\
\hline \multicolumn{3}{|l|}{ 8. Repetição e substituição } \\
\hline 8.3 Formação de hábitos & $\begin{array}{l}\text { Repetição do comportamento no mesmo contexto para que o contexto } \\
\text { provoque o comportamento. }\end{array}$ & $\begin{array}{l}8,13,19,26 \\
29,33,45,51\end{array}$ \\
\hline \multicolumn{3}{|l|}{ 10. Recompensa e ameaça } \\
\hline 10.1 Incentivo material & $\begin{array}{l}\text { Informar que dinheiro, vouchers ou outro objeto de valor será oferecido } \\
\text { se, e só se, existir um esforço e/ou progresso no comportamento. Inclui } \\
\text { reforço positivo. }\end{array}$ & 27,51 \\
\hline 10.3 Incentivo não-específico & $\begin{array}{l}\text { Recompensa se, e só se, houver esforço e/ou progresso no } \\
\text { comportamento. Inclui reforço positivo. }\end{array}$ & $8,24,45,51$ \\
\hline
\end{tabular}


Vários estudos mostram que, a implementação de técnicas de mudança comportamental tem sido eficaz na gestão da doença crónica (Sawyer et al., 2019; Duff et al., 2017). Tal como Mudaliar et al. (2016) referem, a mudança do estilo de vida é um desígnio importante na gestão de risco DCV em pessoas com doença crónica, conduzindo, não apenas a bons resultados em saúde, mas também reforçando a relação custo-efetividade. Os programas implementados conduziram a vários ganhos em saúde, quer sejam cognitivos ou comportamentais, quer sejam em indicadores clínicos (Tabela 2).

Tabela 2. Distribuição dos ganhos em saúde dos participantes, pelos estudos analisados

\begin{tabular}{llcc}
\hline GANHOS EM SAÚDE & & Fi & $\%$ \\
\hline Cognitivos & Conhecimentos adquiridos & 10 & 62.5 \\
\hline Comportamentos & Dieta equilibrada & 6 & 37.5 \\
& Controlo da glicemia & 6 & 37.5 \\
& Prática de exercício físico & 8 & 50 \\
& Auto monitorização & 3 & 18.8 \\
& Adesão ao regime medicamentoso & 3 & 18.8 \\
\hline Indicadores clínicos & Normalização dos valores HbA1c & 6 & 37.5 \\
& Perda de peso & 7 & 43.8 \\
& Normalização do IMC & 5 & 31.3 \\
& Normalização do perímetro & 4 & 25 \\
& cintura/anca & 5 & 31.3 \\
& Controlo da tensão arterial & 6 & 37.5 \\
\hline
\end{tabular}

O período de intervenção nos estudos analisados varia entre 8 semanas a 10 anos, havendo recurso, em grande parte das pesquisas, a ferramentas digitais para implementar a intervenção junto dos participantes (Chao et al., 2017) ou para monitorizar ou complementar a intervenção desenvolvida (Marra et al., 2019; Hung et al., 2016; Koniak-Griffin et al., 2015; Kempf et al., 2017). Alguns estudos têm vido mostrar que as ferramentas digitais têm contribuído não apenas para auto-gestão da doença crónica, como também reduzir as desigualdades em termos de acessibilidade aos serviços de saúde (Wade \& Stocks, 2017).

\section{CONCLUSÕES}

A literatura científica evidencia que os programas de intervenção na gestão do risco de DCV, em pessoas com doença crónica, são basilares para que se obtenham ganhos em saúde. Os principais domínios de intervenção são o exercício físico, alteração dos hábitos alimentares, psicologia motivacional e educação da população sobre os estilos de vida saudáveis, o que pode envolver todas as dimensões preconizadas para cuidar da pessoa com doença crónica. Várias são as técnicas de mudança comportamental mobilizadas pelos autores no desenho dos programas implementados, sendo de maior peso a aposta no melhoramento do conhecimento, na demonstração de comportamentos e na formação de 
hábitos. Nos estudos analisados identificou-se uma diminuição do risco de doenças cardiovasculares associada à sua implementação. Destacam-se como resultados, o aumento da prática de exercício físico, a perda de peso corporal, os conhecimentos de saúde adquiridos e consequentemente o controlo de parâmetros analíticos (glicémia, lipidémia e tensão arterial).

Concluímos que os resultados indicam algumas intervenções promissoras na gestão do risco de DCV em pessoas com doenças crónicas. No entanto, a heterogeneidade dos programas e a inconsistência de alguns resultados impedem conclusões sólidas. É necessário continuar a investigar para tornar os resultados mais consistentes, nomeadamente, importa que os programas a serem desenvolvidos futuramente englobem as componentes como a gestão dos hábitos tabágicos ou alcoólicos e do stress.

\section{REFERÊNCIAS}

Bullard, T., Ji, M., An, R., Trinh, L., Mackenzie, M., \& Mullen, S. P. (2019). A systematic review and meta-analysis of adherence to physical activity interventions among three chronic conditions: cancer, cardiovascular disease, and diabetes. BMC Public Health, 19(1).

Chodosh, J., Morton, S. C., Mojica, W., Maglione, M., Suttorp, M. J., Hilton, L., Rhodes, S., \& Shekelle, P. (2005). Meta-analysis: chronic disease self-management programs for older adults. Annals Of Internal Medicine, 143(6), 427-438.

DGS, Direção-Geral da Saúde de Portugal (2016). A saúde dos Portugueses 2016. Lisboa: DGS.

Duff, O. M., Walsh, D. M., Furlong, B. A., O'Connor, N. E., Moran, K. A., \& Woods, C. B. (2017). Behavior Change Techniques in Physical Activity eHealth Interventions for People With Cardiovascular Disease: Systematic Review. Journal of Medical Internet Research, 19(8), 1. https://doi.org/10.2196/jmir.7782

Higgins JPT, Thomas J, Chandler J, Cumpston M, Li T, Page MJ, Welch VA (editors). Cochrane Handbook for Systematic Reviews of Interventions version 6.0 (updated July 2019). Cochrane, 2019. Available from www.training.cochrane.org/handbook

Kastner, M., Cardoso, R., Lai, Y., Treister, V., Hamid, J. S., Hayden, L., ... \& Holroyd-Leduc, J. (2018). Effectiveness of interventions for managing multiple high-burden chronic diseases in older adults: a systematic review and meta-analysis. Cmaj, 190(34), E1004-E1012.

Luijks $\mathrm{H}$, Lucassen $\mathrm{P}$, van Weel $\mathrm{C}$, et al. How GPs value guidelines applied to patients with multimorbidity: a qualitative study. BMJ Open 2015;5:e007905.

Meader, N., King, K., Llewellyn, A., Norman, G., Brown, J., Rodgers, M., ... \& Stewart, G. (2014). A checklist designed to aid consistency and reproducibility of GRADE assessments: development and pilot validation. Systematic reviews, 3(1), 82.

Michie, S., Atkins, L., \& West, R. (2014). The Behavior Change Wheel: A Guide To Designing Interventions (First edit). London: Silverback Publishing

Michie, S., Richardson, M., Johnston, M., Abraham, C., Francis, J., Hardeman, W., ... \& Wood, C. E. (2013). The behavior change technique taxonomy (v1) of 93 hierarchically clustered techniques: building an international consensus for the reporting of behavior change interventions. Annals of behavioral medicine, 46(1), 81-95.

Moher, D., Shamseer, L., Clarke, M., Ghersi, D., Liberati, A., Petticrew, M., ... \& Stewart, L. A. (2015). Preferred reporting items for systematic review and meta-analysis protocols (PRISMA-P) 2015 statement. Systematic reviews, $4(1), 1$. 
Mudaliar, U., Zabetian, A., Goodman, M., Echouffo-Tcheugui, J. B., Albright, A. L., Gregg, E. W., \& Ali, M. K. (2016). Cardiometabolic Risk Factor Changes Observed in Diabetes Prevention Programs in US Settings: A Systematic Review and Meta-analysis. Plos Medicine, 13(7), e1002095. https://doi.org/10.1371/journal.pmed.1002095

Pengpid, S., Peltzer, K., Puckpinyo, A., \& Chantarasongsuk, I. J. (2019). Effectiveness of a cluster-randomized controlled trial community-based lifestyle intervention program to control prehypertension and/or prediabetes in Thailand. International Journal of Diabetes in Developing Countries, 39(1), 123-131.

Peters, R., Ee, N., Peters, J., Beckett, N., Booth, A., Rockwood, K., \& Anstey, K. J. (2019). Common risk factors for major noncommunicable disease, a systematic overview of reviews and commentary: the implied potential for targeted risk reduction. Therapeutic advances in chronic disease, 10, 2040622319880392.

Reynolds, R., Dennis, S., Hasan, I., Slewa, J., Chen, W., Tian, D., ... \& Zwar, N. (2018). A systematic review of chronic disease management interventions in primary care. BMC family practice, 19(1), 11.

Sawyer, A., Lewthwaite, H., Gucciardi, D. F., Hill, K., Jenkins, S., \& Cavalheri, V. (2019). Behaviour change techniques to optimise participation in physical activity or exercise in adolescents and young adults with chronic cardiorespiratory conditions: a systematic review. Internal Medicine Journal, 49(10), 1209-1220. https://doi.org/10.1111/imj.14141

Uthman, O. A., Hartley, L., Rees, K., Taylor, F., Ebrahim, S., \& Clarke, A. (2015). Multiple risk factor interventions for primary prevention of cardiovascular disease in low-and middle-income countries. Cochrane Database of Systematic Reviews, (8).

Van der Heide, I., Snoeijs, S., Quattrini, S., Struckmann, V., Hujala, A., Schellevis, F., \& Rijken, M. (2018). Patientcenteredness of integrated care programs for people with multimorbidity. Results from the European ICARE4EU project. Health Policy, 122(1), 36-43.

Wade, V., \& Stocks, N. (2017). The Use of Telehealth to Reduce Inequalities in Cardiovascular Outcomes in Australia and New Zealand: A Critical Review. Heart, Lung \& Circulation, 26(4), 331-337. https://doi.org/10.1016/j.hlc.2016.10.013

Wagner, E. H., Austin, B. T., Davis, C., Hindmarsh, M., Schaefer, J., \& Bonomi, A. (2001). Improving chronic illness care: translating evidence into action. Health affairs, 20(6), 64-78.

WHO, World Health Organization (2017). Cardiovascular diseases (CVDs). https://www.who.int/news-room/factsheets/detail/cardiovascular-diseases-(cvds)

WHO, World Health Organization. (2018). Noncommunicable diseases country profiles 2018.

Xu, X., Mishra, G. D., \& Jones, M. (2017). Evidence on multimorbidity from definition to intervention: an overview of systematic reviews. Ageing research reviews, 37, 53-68.

Yeoh, E. K., Wong, M. C. S., Wong, E. L. Y., Yam, C., Poon, C. M., Chung, R. Y., Chong, M., Fang, Y., Wang, H. H. X., Liang, M., Cheung, W. W. L., Chan, C. H., Zee, B., \& Coats, A. J. S. (2018). Benefits and limitations of implementing Chronic Care Model (CCM) in primary care programs: A systematic review. International Journal Of Cardiology, 258, 279-288. https://doi.org/10.1016/j.ijcard.2017.11.057 